\title{
HYDRAULIC CONDUCTIVITY OF A SUSPENSION-AN INVERSE PROBLEM
}

\author{
JIRI MLS \& MARKETA SEDLACKOVA \\ Charles University, Prague, Czech Republic.
}

\begin{abstract}
Transport phenomena in two-phase systems are studied when solving various problems problems of natural science or technology. One of the consti relations defined in porous media mechanics is the relation between the solid phase concentration and the hydraulic conductivity of a two-phase system. Forty-five experiments were carried out with a suspension consisting of water and kaolin. The one-dimensional equations of Darcian mechanics were applied to formulate the studied process mathematically. The nature of the process allowed for finding a solution to the forward problem. Fitting the theoretical solution to the experimental data made it possible to invert the problem and to get the sought hydraulic conductivity as a function of the suspension concentration. The method and the obtained results are presented and discussed.

Key words: porous-media mechanics, hydraulic conductivity, monotonous process, kaolin, solid-phase concentration
\end{abstract}

\section{INTRODUCTION}

In this paper we are concerned with problems of porous media mechanics where both involved phases, the solid phase and the liquid phase, move with their own velocities. The theoretical part of this study is based on equations of dynamics in porous media published by Ref. [1]. The theory is formulated in a set of equations that are presented in the next section. When solving a forward problem, the number of unknown functions that the theory involves exceeds the number of equations and consequently, several equations are required in order to make a complete system. These equations determine hydromechanical characteristics of the particular porous medium and are known as constitutive equations. The number of them depends on the number of space dimensions of the solved problem. It was shown that precisely two constitutive relations are required if the problem is one-dimensional: the relation between solid-phase stress and porosity of the porous medium and the relation between its porosity (or solid-phase concentration) and hydraulic conductivity. Determination of both characteristics has already been studied in papers Refs. [2] and [3]. Our present study revisits the problem of determining the relationship between hydraulic conductivity and the solid-phase concentration of a clay suspension.

Solutions of such problems always precede solutions of forward problems, unless the constitutive relationships have already been known. It may happen that the sought characteristic is difficult to measure. Since this is our case, we utilize the general theory as a tool that makes it possible to get the sought relationship making use of data of another characteristic, which is easier to measure.

The process of a suspension dewatering by means of gravity compression, which can be met in industrial thickeners, serves as an example of a problem that can be solved utilizing the theory with the sought relationship. Generally, the theory is particularly suitable for solving such two-phase (or multiphase) flow problems where the phases move with different velocities and affect the motion of each other.

The data utilized in our study originate from a set of laboratory measurements that were carried out at Charles University in Prague with suspensions of kaolin and water and that 
were also used by Ref. [2]. Suspensions and their sedimentation are not the subject of this paper. These problems were studied elsewhere, see e.g. Refs. [4-7], also for references. The theoretical base of those studies is the theory of mixtures.

Another theoretical approach is used in Refs. [8] and [9]. In these papers, the authors formulate the problem of particle aggregation by means of the population-balance modelling and utilize the computational fluid dynamics to get the solution. We rather make reference to other publications, namely Refs. [10-12], since our measurements and their use require understanding of the suspension structure and specific attention to the degree of coagulation of the kaolin suspension and to the gel point.

In this paper, we take into account the knowledge of the gel point that was suggested by [3]. This enables us to check the utilized data with respect to the assumption of free sedimentation and to enhance the reliability of the obtained results. The above-presented theory is utilized in order to get the sought relation, which is difficult to measure, making use of data that are easy to measure. The achieved results are presented and discussed.

\section{THE UTILIZED THEORY}

We adopt the equations of dynamics of two-phase systems, see Ref. [1], since we study a problem where both the involved phases move with their own velocities. Assuming a onedimensional problem, the governing equations are: the continuity equations of the liquid and solid phase

$$
\begin{aligned}
& \frac{\partial n}{\partial t}(\mathrm{t}, \mathrm{x})+\frac{\partial w}{\partial x}(\mathrm{t}, \mathrm{x})=0 \\
& \frac{\partial n}{\partial t}(\mathrm{t}, \mathrm{x})-\frac{\partial v}{\partial x}(\mathrm{t}, \mathrm{x})=0,
\end{aligned}
$$

and the equations of motion of the liquid and solid phase

$$
\begin{gathered}
\frac{\partial w}{\partial t}(\mathrm{t}, \mathrm{x})+\mathrm{gn}(\mathrm{t}, \mathrm{x})+\frac{n(\mathrm{t}, \mathrm{x})}{\rho_{w}} \frac{\partial p}{\partial x}+\frac{g n(\mathrm{t}, \mathrm{x})}{K(\mathrm{t}, \mathrm{x})} u(\mathrm{t}, \mathrm{x})=0 \\
\frac{\partial v}{\partial t}(\mathrm{t}, \mathrm{x})+\mathrm{g}(1-n(\mathrm{t}-\mathrm{x}))-\frac{1}{\rho_{s}} \frac{\partial \tau}{\partial x}(\mathrm{t}, \mathrm{x})+\frac{1-n(\mathrm{t}-\mathrm{x})}{\rho_{s}} \frac{\partial p}{\partial x}(\mathrm{t}, \mathrm{x})+\frac{g \rho_{w} n(\mathrm{t}, \mathrm{x})}{\rho_{s} K(\mathrm{t}, \mathrm{x})} u(\mathrm{t}, \mathrm{x})=0
\end{gathered}
$$

where $t$ is time, $x$ is space coordinate measured vertically upwards, $w$ and $v$ are the liquid-phase and the solid-phase volumetric flux densities, $g$ is gravity acceleration, $n$ is porosity, $\rho_{w}$ is density of the liquid phase, $\rho_{s}$ is density of the solid phase, $p$ is the liquid-phase pressure, $K$ is hydraulic conductivity of the suspension, $\tau$ is the solid-phase stress and $\mathrm{u}$ is the relative liquid-phase volumetric flux density, i.e. volumetric flux density of the liquid phase relative to the solid phase. These quantities are defined by Ref. [1], we only note here that, as usual, the liquid-phase pressure is related to unit surface of the liquid phase and the solid-phase stress is related to unit surface of the whole two-phase system. The volumetric flux densities of both phases are given as the flux of the phase through unit surface of the whole porous medium. Since the motion of the two-phase system is defined by volumetric flux densities $w$ and $v$, the relative flux density is not independent. It can be expressed in the form.

$$
u=w-\frac{n}{1-n} v
$$


We also define the solid-phase concentration $\mathrm{c}$ as the solid-phase mass contained in unit volume of the two-phase system, i.e.

$$
c=\rho_{s}(1-\mathrm{n})
$$

The steady state form of eqn (3) is Darcy's law. Equations (3) and (4) involve the inertial forces and, unlike equations based purely on Darcy's law, do not require any correction (e.g. introducing the relaxation time) when solving evolutionary problems; for more details see Ref. [13].

When solving a forward problem, the system (1) to (4) can be considered as a system of four equations with four unknown functions: $\mathrm{w}, v, n$ and $p$. In this case, the functions $K$ and $\tau$ are superfluous dependent quantities and two constitutive relations are required in order to get a complete system. Reference [2] presents a set of experimental measurements and a method of utilizing the results in order to determine hydraulic conductivity as a function of porosity. Reference [3] solves the other problem and determines the solid-phase stress as a function of the solid-phase concentration. They also find the value of solid-phase concentration that corresponds to the gel point.

These results enabled us to find experiments, which did not start with a period of free sedimentation and to exclude them from the utilized set. Subsequently we repeated the solution and improve the resulting function: the dependence of hydraulic conductivity on solid-phase concentration.

\section{THE APPLIED MATERIAL AND THE LABORATORY EXPERIMENTS}

Fifty-six laboratory experiments with a two-phase mixture of water and kaolin were carried out at Charles University. In order to make it possible to repeat the measurements, the Zettlitz Ia kaolin was chosen for the solid-phase component since it is a well-defined standard material. The utilized water was deionized before its use in suspensions.

The chemical composition of the utilized kaolin, given in mass percents, is presented in Table 1. A more detailed description of the material can be found in Ref. [2].

Zettlitz Ia kaolin is not a raw material. Since certain ability to coagulate is required, the kaolin is enriched with calcium and sodium cations. However, the coagulation that results from this enrichment is not sufficient to create a sharp and clearly visible interface that is necessary for reliable measurements of its position. In order to get a sufficiently sharp interface, an additional coagulant was applied. Calcium chloride was chosen for this purpose in the rate 4 grams of calcium chloride per $1 \mathrm{~kg}$ of solid phase. This rate was kept fixed for every suspension regardless of its concentration.

Kaolinite is a layered material built up of tetrahedral sheets of $\mathrm{SiO}_{4}$ and octahedral sheets of $\mathrm{Al}_{2} \mathrm{O}_{3}$ that are linked to each other by oxygen atoms, [14]. In water environment, the linkage can be disconnected and groups of $\mathrm{Al}-\mathrm{OH}$ and $\mathrm{Si}-\mathrm{OH}$ arise. Under acidic conditions, the edges carry positive charge whilst the surfaces are negative and the faces and edges attract each other. In this way, a face-to-edge coagulum arises that is known as

Table 1: Composition of Zettlitz Ia kaolin. The values are given in \% of mass. Loss on ignition is $14,02 \%$

\begin{tabular}{llllllll}
\hline $\mathrm{SiO} 2$ & $\mathrm{Al}_{2} \mathrm{Os}$ & $\mathrm{Fe} 2 \mathrm{O} 3$ & $\mathrm{TiO} 2$ & $\mathrm{CaO}$ & $\mathrm{MgO}$ & $\mathrm{K} 2 \mathrm{O}$ & $\mathrm{Na}_{2} \mathrm{O}$ \\
\hline 47,28 & 35,78 & 0,97 & 0,26 & 0,29 & 0,23 & 1,05 & 0,03 \\
\hline
\end{tabular}


card-house coagulum, [12]. This is the coagulum we investigate in this study, since the $\mathrm{pH}$ of the suspension we worked with ranged from 8.5 to 6.2.

Vertical cylinders were used to carry out the laboratory experiments. The height of the cylinders was 2 meters, their inner diameter was $104 \mathrm{~mm}$ and their bottom was impervious. In order to be able to exclude a possible influence of the cylinders' diameter on the measured data, several experiments were repeated with cylinders of diameters 94 and $114 \mathrm{~mm}$.

The suspension of chosen concentration $c_{0}$ was prepared in a separate vessel and then poured in the measuring cylinder. We assume that each experiment starts from a homogeneous state of the suspension column determined by its initial concentration $c_{0}$ and its initial height $L$. Due to their density, the solid-phase particles of the suspension start to move and build a zone of sedimentation. At the beginning, this zone covers all the height of the suspension column. In view of this, the initial conditions of the experiment are

$$
c(0, x)=c_{0} \quad \text { and } \quad w(0, x)=0 \text { for } \quad x \in(0, L)
$$

As a result of the particles motion, a visible interface arises in the upper part of the suspension column. The interface separates suspension from the overlying layer of water. Let us denote by $Y(t)$ the height of the interface above the cylinder's bottom at time $t$. Since the particles that reach the bottom are stopped there, a zone of thickening starts to develop in the lower part of the column, and another interface arises at the bottom. It moves upwards and separates the zone of free sedimentation from the underlying zone of thickening, i.e. zone of $c>c_{0}$. In view of this, the position $Z(t)$ of the lower interface at time $t$ is defined by the equation

$$
Z(t)=\sup \left\{x \geq 0 ; c(t, x)>c_{0}\right\}, \quad t>0
$$

The positions and motion of the interfaces are shown in the Fig. 1, where (u.i.) and (1.i.) stand for the upper interface and lower interface, respectively.

Since the upper interface is visible, it is possible to determine its height $Y(t)$ at time $t$. We carried out 56 such experiments (batch tests) and obtained 56 finite sequences

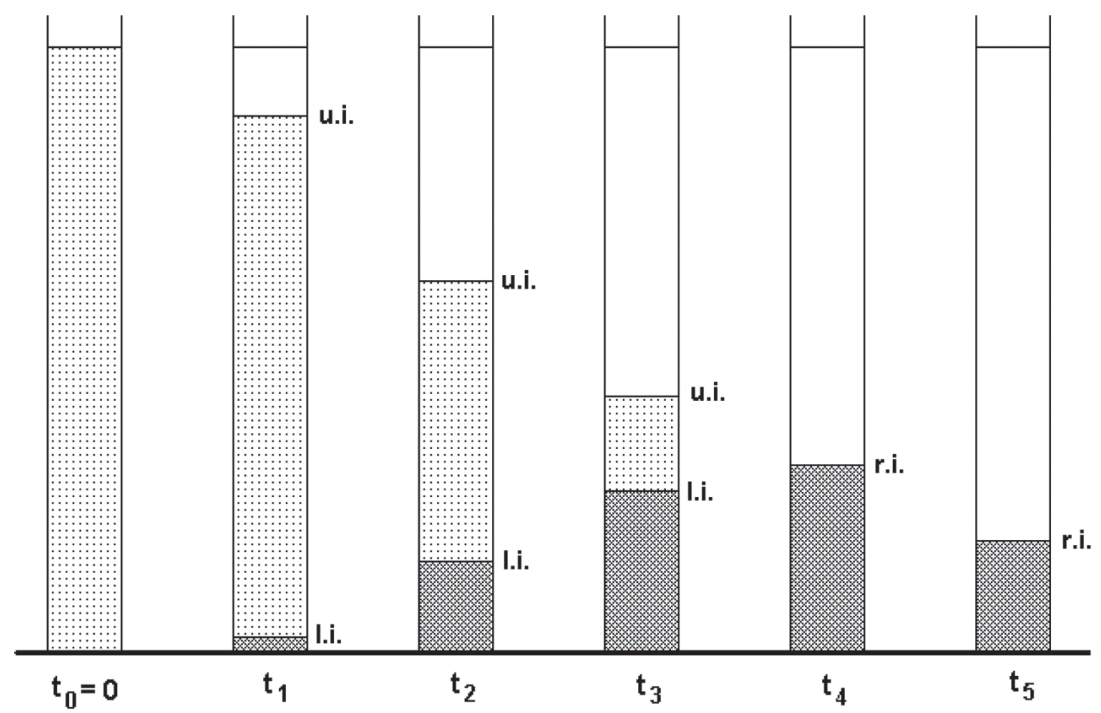

Figure 1: Development of the sedimentation and the compression zones during the experiments 


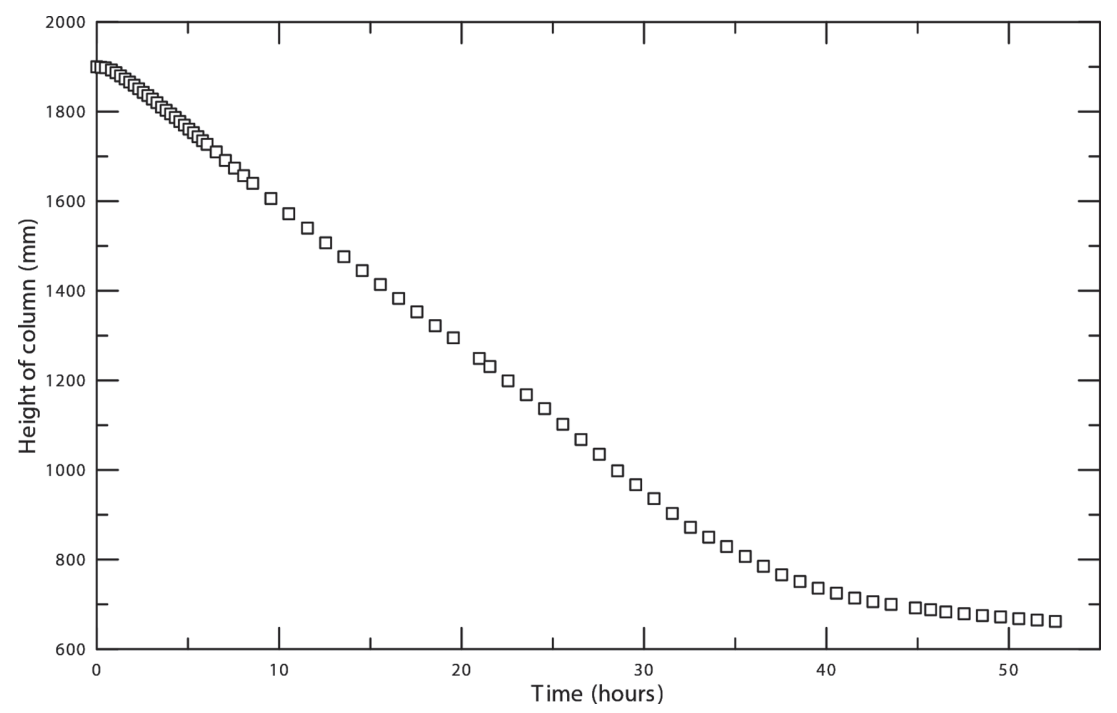

Figure 2: Position of the upper interface above the cylinder's bottom

$$
\left\{\left(t_{n}, y_{n}\right)\right\}_{n=0}^{N_{j}} \quad j=1,2 \ldots, 56
$$

where $y_{n}=Y\left(t_{\mathrm{n}}\right)$ and $N_{j}$ is number of readings in the $j$-th experiment. A beginning part of such a sequence (of the 6-th test) is depicted in Fig. 2.

\section{THE CORRESPONDING FORWARD PROBLEM}

Equations (1) and (2) give

$$
\frac{\partial(w+v)}{\partial x}(t, x)=0
$$

and hence and from the condition of impervious bottom, it follows

$$
w(t, x)=-v(t, x)
$$

Since it holds

$$
\lim _{t \rightarrow 0^{+}} Y(\mathrm{t})=\mathrm{L} \quad \text { and } \quad \lim _{t \rightarrow 0^{+}} Z(\mathrm{t})=0
$$

the set $\Omega$ defined by

$$
\Omega=\left\{(t, x) \in R^{2} ; t>0, x \in(Z(t), Y(t))\right\}
$$

is nonempty and the equality

$$
c(t, x)=c_{0}
$$

holds for every $(t, x) \in \Omega$. In virtue of the last equation and eqn (1), function $w$ is a function of time only in $\Omega$. Taking into account that the solid-phase particle are not in mutual contact in the zone of free sedimentation, we get $\tau=0$ in $\Omega$. According to these results, eqns (3) and (4) give the following ordinary differential equation 


$$
\frac{\mathrm{d} w}{\mathrm{~d} t}+\frac{g \rho_{w} n_{0}}{\left(1-n_{0}\right) k\left(c_{0}\right)\left(\rho_{\mathrm{w}}+n_{0}\left(\rho_{s}-\rho_{w}\right)\right)} w=\frac{g\left(\rho_{\mathrm{s}}-\rho_{\mathrm{w}}\right) n_{0}\left(1-n_{0}\right)}{\rho_{w}+n_{0}\left(\rho_{\mathrm{s}}-\rho_{w}\right)}
$$

where $n_{0}$ is the initial value of porosity related to the initial concentration $c_{0}$ by eqn (6). According to the initial condition $w(0)=0$, the solution to the problem is

$$
w(t)=\rho\left(1-n_{0}\right)^{2} K\left(c_{0}\right)\left(1-\exp \left(\frac{-g n_{0} t}{\left(1-n_{0}\right)\left(1+\rho n_{0}\right) K\left(c_{0}\right)}\right)\right)
$$

where

$$
\rho=\frac{\rho_{s}-\rho_{w}}{\rho_{w}}
$$

We denote by $V(\mathrm{t})$ velocity of the solid-phase particles in domain $\Omega$. Then it holds

$$
V(t)=\frac{\mathrm{d} Y}{\mathrm{~d} t}(t) \quad \text { and } \quad v(t)=\left(1-n_{0}\right) V(t)
$$

The last equations and eqn (16) enable us to formulate the following ordinary differential equation giving the height of the upper interface

$$
\frac{\mathrm{d} Y}{\mathrm{~d} t}=-\rho\left(1-n_{0}\right) K\left(c_{0}\right)\left(1-\exp \left(\frac{-g n_{0} t}{\left(1-n_{0}\right)\left(1+\rho n_{0}\right) K\left(c_{0}\right)}\right)\right)
$$

The solution to this equation that satisfies the initial condition $Y(0)=L$ is

$$
\begin{aligned}
& Y(t)=L-\rho(1-n) K\left(c_{0}\right) t \\
& +\frac{\left(1-n_{0}\right)^{2}\left(1+\rho n_{0}\right) \rho K^{2}\left(c_{0}\right)}{g n_{0}}\left(1-\exp \frac{-g n_{0} t}{\left(1-n_{0}\right)\left(1+\rho n_{0}\right) K\left(c_{0}\right)}\right)
\end{aligned}
$$

\section{FUNCTION $K(C)$}

The solution $Y(\mathrm{t})$ presented in eqn (20) simulates, in the frame of the above introduced theory, the measured height of the upper interface above the cylinder bottom in every experiment. While all the parameters $L, \rho$ and $c_{\mathrm{o}}$ are measurable and known, the value $K\left(c_{\mathrm{o}}\right)$ is unknown and difficult to measure.

Reference [3] determined the value $c_{\mathrm{g}}$ of solid-phase concentration that corresponds to the gel point for the applied suspension: $c_{g}=247 \mathrm{~kg} / \mathrm{m}^{3}$. Making use of this knowledge, we were able to exclude those experiments, in which the condition of starting with free sedimentation was not satisfied. For every experiment such an index $N$ was found, that $t_{N}$ satisfies

$$
t_{N} \leq \sup \{t>0 ; Z(t)<Y(t)\}
$$

Subsequently, the value $K\left(c_{\mathrm{o}}\right)$ was found by means of the least squares method, i.e. by minimizing the sum

where

$$
s=\sum_{i=1}^{N}\left(y_{i}-Y\left(t_{i}\right)\right)^{2}=\sum_{i=1}^{N}\left[\zeta_{i}-A K\left(c_{0}\right) t_{i}+B\left(K\left(c_{0}\right)\right)^{2}\left(1-e_{i}\right)\right]^{2}
$$

$$
\zeta_{i}=L-y_{i}, A=\rho\left(1-n_{0}\right), B=\frac{\left(1-n_{0}\right)^{2}\left(1+\rho n_{0}\right) \rho}{g n_{0}}, e_{i}=\exp \frac{-A t_{i}}{B K\left(c_{0}\right)}
$$


The resulting equations are nonlinear and their roots were found by the bisection method. It was proved that the solutions are unique in the domain of $K>0$. After excluding the outliers, 32 minimizing problems (22) were solved and the set of results

$$
\left\{\left(c_{i}, K_{i}\right)\right\}_{i=1}^{32}
$$

was determined, where the simpler notation $c_{i}$ and $K_{i}$ was introduced instead of $\left(c_{0}\right)_{i}$ and $\left(K\left(c_{0}\right)\right)_{i}$, respectively. It was found, analyzing the obtained data, that hydraulic conductivity decreases with concentration and that a power form of the sought function $K(c)$ can be expected. Making again use of the least squares method, this time for logarithm of values $K_{i}$ and $K\left(c_{i}\right)$, it was found

$$
K(c)=\alpha c^{\beta}
$$

where $c \in[4.5,247], \alpha=7.058 \times 10^{-1}$ and $\beta=-1.992$. The function $K(c)$ and the data (23) are depicted in Fig. 3. It was further found that similarly as the results presented by Ref. [3], the data (23) change their nature from a power form for lower values of concentration to an exponential form for its higher values. The value $c_{c}=90.644 \mathrm{~kg} / \mathrm{m}^{3}$ was found such that

$$
K(c)=a c^{b}, c \in\left[4.5, c_{c}\right]
$$

where $a=4.386 \times 10^{-1}$ and $\mathrm{b}=-1.85169$ and

$$
K(c)=A B^{c}, c \in\left[c_{c}, 247\right]
$$

where $\mathrm{A}=6.635 \times 10^{-4}$ and $\mathrm{B}=9.798 \times 10^{-1}$. Function $K(c)$ defined for $c \in[4.5,247]$ by eqns (25) and (26) is continuous including its derivative in the domain [4.5, 247]. The function is depicted in Fig. 4.

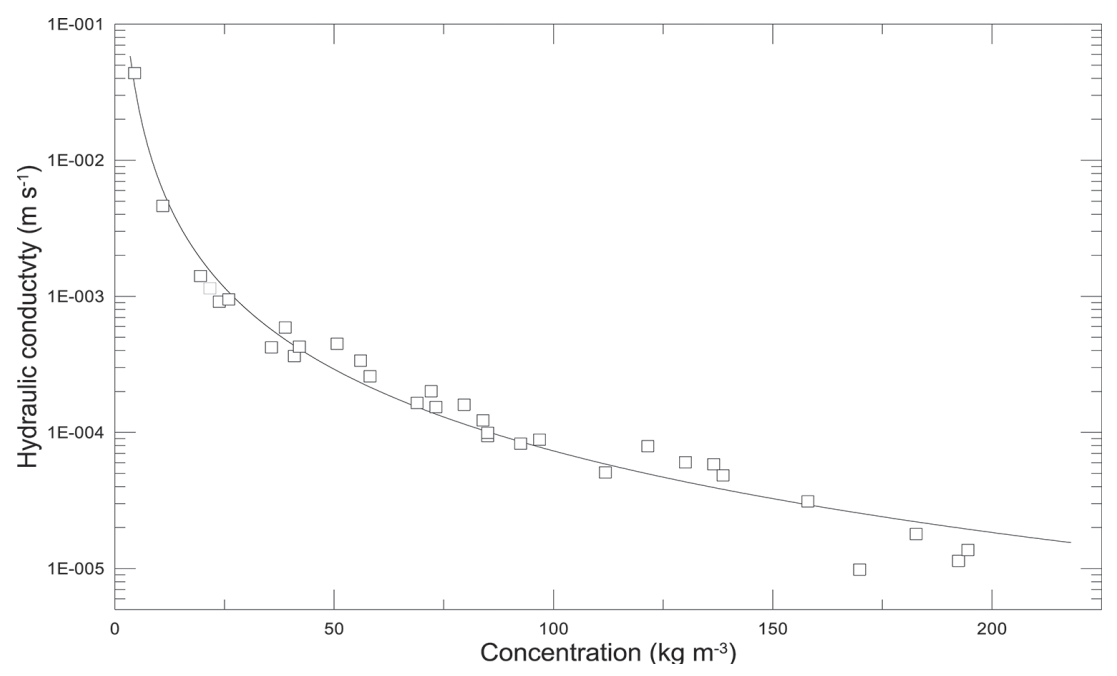

Figure 3: Function $K(c)$ and the computed data $\left(c_{i}, K_{i}\right) i=1, \ldots, 32$ 


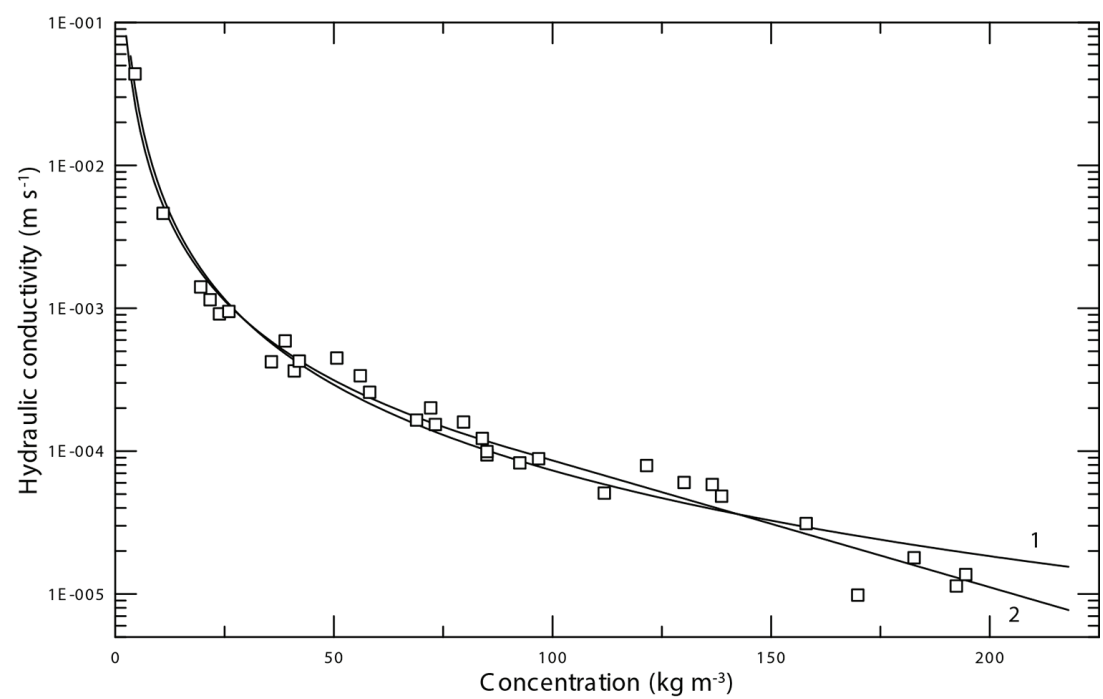

Figure 4: Function $\mathrm{K}(\mathrm{c})$ and the computed data $\left(c_{i}, K_{i}\right) i=1, \ldots, 32$. Curve 1: $K$ according to eqn (24), Curve 2: $K$ according to eqns (25) and (26).

\section{CONCLUSIONS}

The constitutive relationship between solid-phase concentration of a kaolin suspension and its hydraulic conductivity was determined. A set of laboratory experiments was carried out using a well-defined material. During the experiments, couples $\left(t_{i}, y_{i}\right)$ of time and height of suspension column were registered. Since the sought relationship is difficult to measure, the equations of dynamics in porous media, eqns (1)-(4), were utilized in order to formulate and solve such a direct problem that connects the sought characteristic with the measured data. This enabled us to get the sought function $K(c)$ that expresses the dependence of the hydraulic conductivity on the solid-phase concentration. We present two different solutions, function (24) and functions (25) and (26). The latter one changes its nature from a power form for lower concentrations to an exponential form for higher concentrations and fits the experimental data better than the first function, which is a power function.

\section{REFERENCES}

[1] Mls, J., A continuum approach to two-phase porous media. Transport in Porous Media, 35, pp. 15-36, 1999. https://doi.org/10.1023/a:1006508810941

[2] Petrova, M. \& Mls, J., Determination of hydraulic conductivity of a suspension. Journal of Hydrology and Hydromechanics, 61, pp. 134-139, 2013. https://doi.org/10.2478/johh-2013-0018

[3] Mls, J. \& Sedlackova, M., An inverse problem in the Darcian mechanics of porous media. submitted to Transport in Porous Media, 2017.

[4] Betancourt, F., Concha, F. \& Sbarbaro, D., Simple mass balance controllers for continuous sedimentation. Computers \& Chemical Engineering, 54, pp. 34-43, 2013. https://doi.org/10.1016/j.compchemeng.2013.03.014 
[5] Coe, H. S. \& Clevenger, G.H., Methods for determining the capacity of slime-settling tanks. AIME Transactions, 55, pp. 356-384, 1916.

[6] Concha, F., Rulyov, N.N. \& Laskowski, J.S., Settling velocities of particulate systems 18: solid flux density determination by ultra-flocculation. International Journal of Mineral Processing, 104, pp. 53-57, 2012.

https://doi.org/10.1016/j.minpro.2011.12.007

[7] Diehl, S., Zambrano, J. \& Carlsson, B., Steady-state analysis of activated sludge processes with a settler model including sludge compression. Water Research, 88, pp. 104-116, 2016. https://doi.org/10.1016/j.watres.2015.09.052

[8] Gheshlaghi, M.E., Goharrizi, A.S. \& Shahrivar, A.A., Simulation of a semi-industrial pilot plant thickener using CFD approach. International Journal of Mining Science and Technology, 23(1), pp. 63-68, 2013.

https://doi.org/10.1016/j.ijmst.2013.01.010

[9] Gheshlaghi, M.E., Soltani Goharrizi, A., Aghajani Shahrivar, A. \& Abdollahi, H., Modeling industrial thickener using computational fluid dynamics (CFD), a case study: Tailing thickener in the sarcheshmeh copper mine. International Journal of Mining Science and Technology, 23(6), pp. 885-892, 2013.

https://doi.org/10.1016/j.ijmst.2013.11.002

[10] Nasser, M.S. \& James, A.E., Numerical simulation of the continuous thickening of flocculated kaolinite suspensions. International Journal of Mineral Processing, 84(1-4), pp. 144-156, 2007.

https://doi.org/10.1016/j.minpro.2007.05.005

[11] Usher, S.P. \& Scales, P.J., Steady state thickener modelling from the compressive yield stress and hindered settling function. Chemical Engineering Journal, 111(2-3, SI), pp. 253-261, 2005. https://doi.org/10.1016/j.cej.2005.02.015

[12] Satava, V., Theory of ceramic casting-slips liquefaction and optimization of the casting process. Skldf a keramik, 23, pp. 195-199, 1973.

[13] Mls, J. \& Herrmann, L., Oscillations for an equation arising in groundwater flow with the relaxation time. Mathematical Modelling and Analysis, 16(4), pp. 527-536, 2011. https://doi.org/10.3846/13926292.2011.627597

[14] van Olphen, H., An introduction to clay colloid chemistry. Inter-science, New York, 1963. 